IJMMS 30:10 (2002) 627-635

PII. S0161171202007536

http://ijmms.hindawi.com

(c) Hindawi Publishing Corp.

\title{
COINCIDENCES AND FIXED POINTS OF RECIPROCALLY CONTINUOUS AND COMPATIBLE HYBRID MAPS
}

\author{
S. L. SINGH and S. N. MISHRA
}

Received 16 February 2001 and in revised form 27 March 2001

\begin{abstract}
It is proved that a pair of reciprocally continuous and nonvacuously compatible singlevalued and multivalued maps on a metric space possesses a coincidence. Besides addressing two historical problems in fixed point theory, this result is applied to obtain new general coincidence and fixed point theorems for single-valued and multivalued maps on metric spaces under tight minimal conditions.
\end{abstract}

2000 Mathematics Subject Classification: 47H10.

1. Introduction. The concept of compatible maps has proven useful for generalizing results in the context of metric fixed point theory for continuous single-valued and multivalued maps (cf. [1, 2, 4, 11, 15, 16, 20, 21, 22, 23, 30, 31]). Recently, reciprocal continuity for a pair of (discontinuous) single-valued maps has been introduced in [23] and promoted as a means to comprehensive results.

First, we introduce reciprocal continuity for a hybrid pair of single-valued and multivalued maps, and emulate the joint merits of reciprocal continuity and compatibility of a hybrid pair in the setting of metric spaces. We give a general principle (Theorem 2.8) stating that nonvacuously compatible and reciprocally continuous hybrid pair on a metric space has a coincidence. This seems to be of vital interest in view of a historically significant and negatively settled problem that a pair of continuous and commuting self-maps on the closed interval $[0,1]$ has a common fixed point (see $[3,13,17])$ and that continuous and commuting maps on a complete metric space need not have a coincidence even (see Remark 2.7(v)). This principle presents another view of a significant result of Jungck [17, Theorem 3.6] on a metric space as well. We apply Theorem 2.8 to obtain a coincidence and fixed point theorem for a hybrid quadruple of maps on a metric space satisfying a very general contractive type condition which includes several general conditions studied by Beg and Azam [1], Ćirić [6], Das and Naik [10], Jungck [16], Kaneko [19, 20], Rhoades et al. [27], Singh et al. [29], Tan and Minh [33], and others. One of our results presents another view of recent resolutions to a still open fixed point problem of Simon Reich (see $[4,5,8,9,25,26]$ ). Our final result on a compact metric space extends and generalizes fixed point theorems from $[7,17,32,33]$.

In this paper, consistent with [22, page 620], $(X, d)$ denotes a metric space, id the identity map on $X, \mathrm{CL}(X)$ (resp., $\mathrm{CB}(X))$ the nonempty closed (resp., closed and bounded) subsets of $X$ and $H$ for the Hausdorff (resp., generalized Hausdorff) metric on $\mathrm{CB}(X)$ (resp., $\mathrm{CL}(X)$ ). Further, $d(A, B)$ denotes the ordinary distance between 
nonempty subsets $A$ and $B$ of $X$ while $d(x, B)$ stands for $d(A, B)$ when $A=\{x\}$. The set of natural numbers is denoted by $\mathbb{N}$.

\section{Reciprocal continuity}

Definition 2.1. The maps $T: X \rightarrow \mathrm{CL}(X)$ and $f: X \rightarrow X$ are reciprocally continuous on $X$ (resp., at $t \in X$ ) if and only if $f T x \in \operatorname{CL}(X)$ for each $x \in X$ (resp., $f T t \in \mathrm{CL}(X))$ and $\lim _{n} f T x_{n}=f M, \lim _{n} T f x_{n}=T t$ whenever $\left\{x_{n}\right\}$ is a sequence in $X$ such that

$$
\lim _{n} T x_{n}=M \in \mathrm{CL}(X), \quad \lim _{n} f x_{n}=t \in M .
$$

We may use r.c. for "reciprocal continuity" or "reciprocal continuous" as the situation demands. For self-maps $f, g: X \rightarrow X$, this definition due to Pant [23] reads: $f$ and $g$ are r.c. if and only if $\lim _{n} g f x_{n}=g t$ and $\lim _{n} f g x_{n}=f t$ whenever $\left\{x_{n}\right\} \subset X$ is such that $\lim _{n} g x_{n}=\lim _{n} f x_{n}=t \in X$. Clearly, any continuous pair is reciprocally continuous but, as the following examples show, the converse is not true.

EXAMPLE 2.2 (see [24, Example 2.1]). Let $X=[0, \infty)$ with the usual metric. Define $g, f: X \rightarrow X$ by

$$
g x=\left\{\begin{array}{ll}
\frac{1}{2}+x & \text { if } 0 \leq x<\frac{1}{2}, \\
1 & \text { if } x=\frac{1}{2}, \\
0 & \text { if } x>\frac{1}{2},
\end{array} \quad f x= \begin{cases}\frac{1}{2}-x & \text { if } 0 \leq x<\frac{1}{2}, \\
\frac{1}{2} & \text { if } x=\frac{1}{2}, \\
1 & \text { if } x>\frac{1}{2} .\end{cases}\right.
$$

These maps are discontinuous at $x=1 / 2$. However, they are r.c. (take a decreasing sequence $\left\{x_{n}\right\}$ converging to 0 ).

EXAMPLE 2.3. Let $X=R^{1}$,

$$
T X=\left\{\begin{array}{ll}
{\left[\frac{1}{2}, x+1\right]} & \text { if } x>0, \\
\{0\} & \text { if } x=0, \\
{\left[x-1,-\frac{1}{2}\right]} & \text { if } x<0,
\end{array} \quad f x= \begin{cases}2 x+1 & \text { if } x>0, \\
0 & \text { if } x=0, \\
2 x-1 & \text { if } x<0 .\end{cases}\right.
$$

Then $T$ and $f$ are r.c. at $x=0$ (take $x_{n}=0, n \in \mathbb{N}$ ). Notice that there is a discontinuity at their common fixed point $(x=0)$.

For continuity of multivalued maps at their fixed and common fixed points, refer to [12]. The following definition is due to Kaneko and Sessa [20] and Beg and Azam [1] when $T: X \rightarrow \mathrm{CB}(X)$.

Definition 2.4. The maps $T: X \rightarrow \mathrm{CL}(X)$ and $f: X \rightarrow X$ are compatible if and only if $f T x \in \mathrm{CL}(X)$ for each $x \in X$ and $\lim _{n} H\left(T f x_{n}, f T x_{n}\right)=0$ whenever $\left\{x_{n}\right\}$ is a sequence in $X$ such that $\lim _{n} T x_{n}=M \in \mathrm{CL}(X)$ and $\lim _{n} f x_{n}=t \in M$.

Evidently commuting maps $T, f$ (i.e., when $f T x=T f x, x \in X$ ) are weakly commuting (i.e., $H(T f x, f T x) \leq d(f x, T x), x \in X$, [19, 29]), weakly commuting $T, f$ are compatible, and compatible $T, f$ are weakly compatible (i.e., when $f T x=T f x$ 
whenever $f x \in T x,[18])$ but the reverse implication is not true. For an excellent discussion on the role of weak compatibility in fixed point considerations, refer to Jungck and Rhoades [18]. For self-maps $f, g: X \rightarrow X$, Definition 2.4 due to Jungck $[15,16]$ reads: $f$ and $g$ are compatible if and only if $\lim _{n} d\left(g f x_{n}, f g x_{n}\right)=0$ wherever $\left\{x_{n}\right\}$ is a sequence in $X$ such that $\lim _{n} f x_{n}=\lim _{n} g x_{n}=t \in X$. Notice that the maps $g, f$ of Example 2.2 are not compatible (take $\left\{x_{n}\right\}$ as in Example 2.2). So r.c. need not imply compatibility. Taking $X$ as in Example 2.2 and defining $f x=3 x^{2} /\left(x^{2}+8\right)$ and $g x=x$, if $x<2, g x=2$ if $x \geq 2$, one may conclude that $f, g$ are r.c. but not compatible (take $x_{n}=4, n \in \mathbb{N}$ ). Hence we assert that: the r.c. and compatibility are independent concepts.

Following Itoh and Takahashi [14], $T: X \rightarrow \mathrm{CL}(X)$ and $f: X \rightarrow X$ are IT-commuting (commuting in the sense of Itoh-Takahashi [14]) at a point $v \in X$ if $T f v \subset f T v$. Further, $T$ and $f$ are IT-commuting on $X$ if they are IT-commuting at each point $v \in X$. We remark that the IT-commutativity of a hybrid pair $(T, f)$ at a point $v$ is more general than its compatibility (cf. [31, Example 1]) and weak compatibility at the point $v$.

EXAMPLE 2.5. Let $X=[0, \infty)$ be endowed with the usual metric and

$$
T x=\left\{\begin{array}{ll}
{[0, x]} & \text { if } x<2, \\
{[4,2+x]} & \text { if } x \geq 2,
\end{array} \quad f x= \begin{cases}x & \text { if } x<2, \\
4 & \text { if } x \geq 2\end{cases}\right.
$$

We see that $T$ and $f$ are compatible for $x<2$ but not for $x \geq 2$ (e.g., take $x_{n}=2+n$, $n \in \mathbb{N}$ ). Further, $T$ and $f$ are not r.c. (e.g., take $x_{n}=2-1 / n, n \in \mathbb{N}$ ).

EXAMPLE 2.6. Let $X=[2, \infty)$ with the usual metric and $T x=\{1+x\}$ and $f x=$ $2 x+1$. We see that there does not exist a sequence $\left\{x_{n}\right\} \subset X$ such that $\left\{f x_{n}\right\}$ and $\left\{T x_{n}\right\}$ both converge to the same element in $X$. Thus requirements of compatibility are vacuously satisfied .

REMARK 2.7. (i) If a compatible pair $(T, f)$ is such that $T t=f M$, then it is evident from Definitions 2.1 and 2.4 that the continuity of one of $T$ or $f$ is sufficient to ensure the r.c. of the pair $(T, f)$.

(ii) If $T$ and $f$ are r.c. and nonvacuously compatible, then $T t=f M$. See the proof of Theorem 2.8.

(iii) The r.c. at a point $t \in X$ may be verified by considering all sequences $\left\{x_{n}\right\} \subset X$ such that $\lim _{n} f x_{n}=t \in M=\lim _{n} T x_{n}$. If there does not exist such a sequence then the definition holds vacuously, and the maps are r.c. (see Example 2.6). This observation applies to the compatibility of $T$ and $f$ as well. Hence nonvacuous compatibility of $T$ and $f$ implies the existence of at least a sequence $\left\{x_{n}\right\}$ in $X$ such that $\left\{f x_{n}\right\}$ and $\left\{T x_{n}\right\}$ both converge as per requirements of Definition 2.4.

(iv) If the pair $(T, f)$ is compatible at a point $v \in X$ and $v$ is a coincidence point of $T$ and $f$, that is, $f v \in T v$, then $f T v=T f v$ (see [20, page 260]). Indeed, commutativity, weak commutativity, and compatibility of $T$ and $f$ are equivalent at a coincidence point $v$ of $T$ and $f$.

(v) A pair of continuous and commuting selfmaps of a complete metric space need not have a coincidence; for example, $g x=1+x$ and $f x=x, x \in[0, \infty)$. (Notice the vacuous compatibility of $g$ and $f$.) 
The following is the main result of this section. In all that follows, $C(T, f)$ stands for the collection of coincidence points of $T$ and $f$, that is, $C(T, f)=\{v: f v \in T v\}$.

THeOrem 2.8. Let $(X, d)$ be a metric space and $T: X \rightarrow \operatorname{CL}(X)$ and $f: X \rightarrow X$. If $T$ and $f$ are reciprocally continuous and nonvacuously compatible on $X$ then $C(T, f)$ is nonempty. Further, $T$ and $f$ have a common fixed point $f t$, provided $f f t=f t$ for some $t \in C(T, f)$.

Proof. Since $T$ and $f$ are nonvacuously compatible, there exits a sequence $\left\{x_{n}\right\}$ in $X$ such that $\left\{f x_{n}\right\}$ and $\left\{T x_{n}\right\}$ converge, respectively, to $t \in X$ and $M \in \operatorname{CL}(X)$ such that $t \in M$ and $\lim _{n} H\left(T f x_{n}, f T x_{n}\right)=0$. This, in view of the r.c. of $T$ and $f$, yields $H(T t, f M)=0$, and $T t=f M$. Now, $t \in M$ implies $f t \in f M$. Therefore $f t \in T t$, and $C(T, f)$ is nonempty. Further, $f t=f f T$ implies $f t \in f T t=T f t$ (cf. Remark 2.7(i)). This completes the proof.

For a better appreciation of Theorem 2.8 and the relative roles of r.c. and compatibility, consider the following result of Mizoguchi and Takahashi [21, Theorem 3].

THEOREM 2.9. Let $K$ be a closed convex subset of a uniformly convex Banach space. Let $T: K \rightarrow \mathrm{CB}(X)$ and $f: K \rightarrow K$ be such that $T x$ is convex for each $x \in K, H(T x, T y) \leq$ $q\|x-y\|, x, y \in K, 0 \leq q<1$, and $\|f x-f p\| \leq\|x-p\|, x \in K, p \in F(f)$, where $F(f)$ denotes the set of fixed points of $f$. If $T$ and $f$ are IT-commuting on $K$, then $T$ and $f$ have a common fixed point, that is, there exists $z \in F(f)$ with $f z \in T z$.

Further, Theorem 2.8 applies to discontinuous maps and a common fixed point may be a point of discontinuity as well (see Example 2.3). Notice that, besides several stronger conditions on the space, $T$ of Theorem 2.9 is a multivalued contraction and $f$ is nonexpansive about fixed points.

3. Results on metric spaces and Reich's problem. First, we give a very general coincidence and fixed point theorem under very tight conditions. Let $\psi$ denote the family of maps $\phi$ from the set $\mathbb{R}^{+}$of nonnegative reals to itself such that $\phi(t)<t$ for all $t>0$.

Theorem 3.1. Let $(X, d)$ be a metric space and $S, T: X \rightarrow \operatorname{CL}(X)$ and $f, g: X \rightarrow X$ such that

(1) $S(X) \subset g(X)$ and the pair $(S, f)$ is reciprocally continuous and nonvacuously compatible.

If there exists $\phi \in \psi$ such that

(2) $H(S x, T y) \leq \phi(M(x, y))$ for $x, y$ in $X$, where

$$
M(x, y)=\max \{d(f x, g y), d(f x, S y), d(g y, T y), d(f x, T y), d(g y, S x)\},
$$

then $C(S, f)$ and $C(T, g)$ are nonempty. Further,

(Ia) $S$ and $f$ have a common fixed point $f t$, provided $f f t=f t$ for some $t \in$ $C(S, t)$;

(Ib) $T$ and $g$ have a common fixed point gu, provided $g g u=g u$ and $T, g$ are IT-commuting at $u \in C(T, g)$; 
(Ic) $S, T, f$, and $g$ have a common fixed point, provided (Ia) and (Ib) both are true.

Proof. By Theorem 2.8, (1) implies that $C(S, f)$ is nonempty, that is, $f t \in S t$ for some $t \in X$. Since $S(X) \subset g(X)$, there is a point $u \in X$ such that $f t=g u \in S t$. So by (2),

$$
\begin{aligned}
d(g u, T u) & \leq H(S t, T u) \\
& \leq \phi(\max \{d(f t, g u), d(f t, S t), d(g u, T u), d(f t, T u), d(g u, S t)\}) \\
& =\phi(d(g u, T u))<d(g u, T u) \quad \text { if } g u \notin T u .
\end{aligned}
$$

So $g u \in T u$ and $C(T, g)$ is nonempty.

(Ia) and (Ib) may be shown following the last part of the proof of Theorem 2.8. Now (Ic) is immediate.

Several contractive conditions studied in $[1,4,6,10,11,12,16,18,19,20,27,28$, 29, 33] are special cases of (2). For example, if we take $\phi(t)=q t(0 \leq q<1), f=g$, $S=T$, and $T: X \rightarrow X$, then we obtain the condition studied in [10]. Notice that the contractive condition studied in [6] is a special case of that of [10].

Reich $[25,26]$ posed the following question: let $(X, d)$ be a complete metric space and $T: X \rightarrow \mathrm{CB}(X)$ such that $H(T x, T y) \leq k(d(x, y)) d(x, y)$ for all distinct $x, y \in$ $X$, where $k:(0: \infty) \rightarrow(0,1)$ with $\lim _{r \rightarrow t^{+}} \sup k(r)<1$ for each $t>0$. Then, does $T$ have a fixed point? Mizoguchi and Takahashi [21] have shown that $T$ has a fixed point when $\lim _{r \rightarrow t^{+}} k(r)<1$ for each $t \geq 0$. Chang [4] has generalized this result, and Theorem 3.1 presents an extension of Chang's main result [4, Theorem 1]. However, Reich's problem remains open and needs further resolution.

The following example shows that the nonvacuous compatibility of one of the pairs $(S, f)$ or $(T, g)$ is essential even if $f=g=\mathrm{id}$.

EXAMPLE 3.2 (see [28]). Let $X=\{1,2,3,4\}$ with metric $d$ defined by

$$
d(1,2)=d(3,4)=2, \quad d(1,3)=d(2,4)=1, \quad d(1,4)=d(2,3)=\frac{3}{2} .
$$

Define $S$ and $T$ by $S 1=S 4=\{2\}, S 2=S 3=\{1\}$ and $T 1=T 3=\{4\}, T 2=T 4=\{3\}$. Take $f=g=i d$. Then Definition 2.4 is satisfied with $\phi(t)=3 t / 4$. All conditions of Theorem 3.1 are satisfied except (1). Indeed the pairs $(S$,id) and $(T$, id) are vacuously compatible.

The following result generalizes several main results from $[1,11,16,18,19,20]$ and others.

COROLlary 3.3. Let $(X, d)$ be a complete metric space and $S, T: X \rightarrow \mathrm{CL}(X), f, g:$ $X \rightarrow X$ such that

(3) $S(X) \subset g(X), T(X) \subset f(X)$, and the pair $(S, f)$ is compatible and reciprocally continuous.

If there exists $q \in(0,1)$ such that 
(4) $H(S x, T y) \leq q m(x, y)$ for $x, y$ in $X$, where

$$
m(x, y)=\max \left\{d(f x, g y), d(f x, S x), d(g y, T y), \frac{1}{2}[d(f x, T y)+d(g y, S x)]\right\},
$$

then $C(S, f)$ and $C(T, g)$ are nonempty. Further, conclusions (Ia), (Ib), and (Ic) are also true.

Proof. Since $S(X) \subset g(X)$ and $T(X) \subset f(X)$, we construct the sequences $\left\{x_{n}\right\}$, $\left\{y_{n}\right\} \subset X$ as in $[11,31]$ such that, for each $n \in \mathbb{N}$,

$$
y_{2 n-1}=g x_{2 n-1} \in S x_{2 n-2}, \quad y_{2 n}=f x_{2 n} \in T x_{2 n-1} .
$$

Then as in [11],

$$
\begin{aligned}
& d\left(y_{2 n-1}, y_{2 n}\right) \leq q^{-1 / 2} d\left(y_{2 n-2}, y_{2 n-1}\right) \\
& d\left(y_{2 n}, y_{2 n+1}\right) \leq q^{-1 / 2} d\left(y_{2 n-1}, y_{2 n}\right)
\end{aligned}
$$

So $\left\{y_{n}\right\}$ converges to a point $t \in X$, [11, Theorem 2]. By (4),

$$
\begin{aligned}
H\left(S x_{2 n}, T x_{2 n-1}\right) & \leq q m\left(x_{2 n}, x_{2 n-1}\right) \\
& \leq q \max \left\{d\left(y_{2 n}, y_{2 n-1}\right), d\left(y_{2 n}, y_{2 n+1}\right)\right\} \rightarrow 0 \text { as } n \rightarrow \infty
\end{aligned}
$$

Therefore, $\lim _{k, n \rightarrow \infty} H\left(S x_{2 n}, S x_{2 k}\right) \leq \lim _{k, n \rightarrow \infty} d\left(y_{2 n-1}, y_{2 k-1}\right)=0$, and $\left\{S x_{2 n}\right\}$ is Cauchy in $\mathrm{CL}(X)$. The space $X$ being complete, the hyper space $\mathrm{CL}(X)$ is also complete, and $\left\{S x_{2 n}\right\}$ converges to an $M$ in $\operatorname{CL}(X)$. So

$$
d(t, M) \leq d\left(t, g x_{2 n-1}\right)+H\left(S x_{2 n-2}, M\right) \longrightarrow 0 \quad \text { as } n \longrightarrow \infty, t \in M .
$$

Thus, for the sequence $\left\{x_{n}\right\}$ in $X$, we have $\left\{S x_{2 n}\right\}$ and $\left\{g x_{2 n-1}\right\}$ converging, respectively, to $M$ and $t \in M$. Therefore the compatibility of the pair $(S, g)$ is nonvacuous. Hence the proof is immediate from Theorem 3.1 by observing that (4) implies (2).

REMARK 3.4. (i) It is evident from the proof of Theorem 3.1 that $f t \in S t$ and $f t=$ $g u \in T u$, that is, $(S, t)$ and $(T, g)$ may have different coincidence points with $f t=g u$. See Example 3.5 in support of this observation, which applies to Corollary 3.3 as well.

(ii) The power of Corollary 3.3 is appreciated by observing that the main result of [11, Theorem 2] is obtained under condition (4) when all the maps $S, T, f, g$ are continuous and the pairs $(S, f)$ and $(T, g)$ are compatible. Moreover, Corollary 3.3 generalizes several other interesting results from $[1,16,19,20]$ and the references therein.

(iii) If $f=$ id under the condition (4) of Corollary 3.3, then the pair $(S, f)$ is automatically compatible and r.c. Thus if $f=g=$ id then Corollary 3.3 states that $S, T: X \rightarrow \mathrm{CL}(X)$, satisfying condition (4), have a common fixed point in complete $X$. 
Therefore, several general results surveyed in [21] are contained in Corollary 3.3 (e.g., Theorems 12, 15, and 16).

EXAMPLE 3.5. Let $X=[0, \infty)$ be endowed with the usual metric. Let $f, g, S, T: X \rightarrow X$ be such that $f x=8 x^{4}, g x=8 x^{8}, S x=x^{4}+7 / 16$, and $T x=x^{8}+7 / 16$. Evidently, for any $x, y \in X, d(S x, T y)=1 / 8 d(f x, g y)$, that is, condition (4) is satisfied with $q=1 / 8$. Further, $S(X)=T(X)=[7 / 16, \infty] \subset X=f(X)=g(X)$, the pair $(S, f)$ is compatible and continuous. So all the hypotheses of Corollary 3.3 are satisfied and $f(1 / 2)=S(1 / 2)$ and $g\left(2^{-1 / 2}\right)=T\left(2^{-1 / 2}\right)$, that is, $f, S$ have a coincidence at $x=1 / 2$, and $g, T$ have a (different) coincidence at $x=\left(2^{-1 / 2}\right)$. Notice that $f(1 / 2)=g\left(2^{-1 / 2}\right)$ and the pair $(T, g)$ is not compatible.

COROLlary 3.6. Let $f, g, S, T$ be self-maps of a complete metric space $(X, d)$ such that $S(X) \subset g(X), T(X) \subset f(X)$, and the pair $(S, f)$ is compatible and reciprocally continuous. If there exists $q \in(0,1)$ such that $d(S x, T y) \leq q m(x, y)$ for all $x, y$ in $X$, then

(IIa) $S$ and $f$ have a common fixed point;

(IIb) $T$ and $g$ have a coincidence at $x=u \in X$;

(IIc) $f, g, S$, and $T$ have a common fixed point provided that $T$ and $g$ are weakly compatible.

The tightness of the conditions in this result is evident from the fact that Jungck's [16, Theorem 3.1] is Corollary 3.6(IIc) with the r.c. replaced by continuity of $f, g$, and the weak compatibility of $T, g$ (at $u \in X$ ) replaced by the compatibility of $T, g$ on $X$.

4. Results on compact spaces. We apply Corollary 3.3 to obtain a new coincidence theorem for a hybrid of multivalued and single-valued maps on the setting of a compact metric space generalizing and extending [7, Theorem 1], [16, Theorem 3.2], and relevant results of Smithson [32] and Tan and Minh [33].

THEOREM 4.1. Let $f, g$ be continuous self-maps of a compact metric space $(X, d)$ and let $S, T: X \rightarrow \mathrm{CB}(X)$ be continuous such that $S(X) \subset g(X), T(X) \subset f(X)$, and the pair $(S, f)$ is compatible. If $H(S x, T y)<m(x, y)$ (see condition (4)) when $m(x, y)>0$, then $C(S, f)$ and $C(T, g)$ are nonempty. Further, (Ia)-(Ic) are also true.

Proof. In view of the conclusions of Corollary 3.3, it is enough to show that $C(S, t)$ and $C(T, g)$ are nonempty. We claim that $m(x, y)=0$ for some $x, y \in X$. Otherwise the function $w(x, y)=H(S x, T y) / m(x, y)$ is continuous and satisfies $w(x, y)<1$ for $(x, y) \in X \times X$. Since $X \times X$ is compact, there exist $v, z \in X$ such that $w(x, y) \leq w(v, z)=q<1$ for $x, y \in X$. Consequently, $H(S x, T y) \leq q m(x, y)$ for $x, y \in X$ and some $q \in(0,1)$. So, by Corollary 3.3 (see also Remark 3.4(i)), there exist $u, t \in X$ such that $f t \in S t$ and $f t=g u \in T u$, and we have $m(t, u)=0$, contradicting $m(t, u)>0$. Thus $m(x, y)=0$ for some $x, y \in X$. Consequently, $f x=g y$, $f x \in S x, g y \in T y$, and this completes the proof.

COROLlary 4.2. Let $f, g, S$, and $T$ be continuous self-maps of a compact metric space $(X, d)$ such that $S(X) \subset g(X), T(X) \subset f(X)$, and the pair $(S, f)$ is compatible. If $d(S x, T y)<m(x, y)$ when $m(x, y)>0$, then conclusions (IIa)-(IIc) are true. 
Its proof may also be completed using Corollary 3.6 and following the proof of Theorem 4.1. Jungck [16, Theorem 3.2] is Corollary 3.6(IIc) when the pair $(T, g)$ is also compatible on $X$.

ACKNOWLEDGMENTS. The authors are highly indebted to the referee for his/her valuable suggestions for improving this paper. They thank Er. Yash Kumar for his work in preparing this paper. The research of the second author was supported by FRD Grant GUN: 2039007.

\section{REFERENCES}

[1] I. Beg and A. Azam, Fixed points of asymptotically regular multivalued mappings, J. Austral. Math. Soc. Ser. A 53 (1992), 313-326.

[2]_, Common fixed points for commuting and compatible maps, Discuss. Math. Differential Incl. 16 (1996), 121-135.

[3] W. M. Boyce, Commuting functions with no common fixed point, Trans. Amer. Math. Soc. 137 (1969), 77-92.

[4] T. H. Chang, Common fixed point theorems for multivalued mappings, Math. Japon. 41 (1995), no. 2, 311-320.

[5] Y. Chen, On a fixed point problem of Reich, Proc. Amer. Math. Soc. 124 (1996), no. 10, 3085-3088.

[6] L. B. Ćirić, A generalization of Banach's contraction principle, Proc. Amer. Math. Soc. 45 (1974), 267-273.

[7] P. Z. Daffer and H. Kaneko, Multi-valued $f$-contractive mappings, Boll. Un. Mat. Ital. A (7) 8 (1994), 233-241.

[8] _ Fixed points of generalized contractive multi-valued mappings, J. Math. Anal. Appl. 192 (1995), 655-666.

[9] P. Z. Daffer, H. Kaneko, and W. Li, On a conjecture of S. Reich, Proc. Amer. Math. Soc. 124 (1996), no. 10, 3159-3162.

[10] K. M. Das and K. V. Naik, Common fixed-point theorems for commuting maps on a metric space, Proc. Amer. Math. Soc. 77 (1979), 369-373.

[11] O. Hadžić, Common fixed point theorems for single-valued and multivalued mappings, Univ. u Novom Sadu Zb. Rad. Prirod.-Mat. Fak. Ser. Mat. 18 (1988), no. 2, 145-151.

[12] T. Hicks and B. E. Rhoades, Fixed points and continuity for multivalued mappings, Int. J. Math. Math. Sci. 15 (1992), 15-30.

[13] J. P. Huneke, On common fixed points of commuting continuous functions on an interval, Trans. Amer. Math. Soc. 139 (1969), 371-381.

[14] S. Itoh and W. Takahashi, Single-valued mappings, multivalued mappings and fixed-point theorems, J. Math. Anal. Appl. 59 (1977), 514-521.

[15] G. Jungck, Compatible mappings and common fixed points, Int. J. Math. Math. Sci. 9 (1986), 771-779.

[16]___ Common fixed points for commuting and compatible maps on compacta, Proc. Amer. Math. Soc. 103 (1988), no. 3, 977-983.

[17] C Common fixed points for compatible maps on the unit interval, Proc. Amer. Math. Soc. 115 (1992), no. 2, 495-499.

[18] G. Jungck and B. E. Rhoades, Fixed points for set valued functions without continuity, Indian J. Pure Appl. Math. 29 (1998), no. 3, 227-238.

[19] H. Kaneko, A common fixed point of weakly commuting multi-valued mappings, Math. Japon. 33 (1988), no. 5, 741-744.

[20] H. Kaneko and S. Sessa, Fixed point theorems for compatible multi-valued and singlevalued mappings, Int. J. Math. Math. Sci. 12 (1989), 257-262.

[21] N. Mizoguchi and W. Takahashi, Fixed point theorems for multivalued mappings on complete metric spaces, J. Math. Anal. Appl. 141 (1989), 177-188. 
[22] S. B. Nadler Jr., Hyperspaces of Sets, Marcel Dekker, New York, 1978.

[23] R. P. Pant, A common fixed point theorem under a new condition, Indian J. Pure Appl. Math. 30 (1999), no. 2, 147-152.

[24] H. K. Pathak, Y. J. Cho, S. M. Kang, and B. Madharia, Compatible mappings of type $(C)$ and common fixed point theorems of Greguš type, Demonstratio Math. 31 (1998), no. 3, 499-518.

[25] S. Reich, Some fixed point problems, Atti Accad. Naz. Lincei Rend. Cl. Sci. Fis. Mat. Natur. (8) 57 (1974), 194-198.

[26] _ Some problems and results in fixed point theory, Topological Methods in Nonlinear Functional Analysis (Toronto, Ont., 1982), Comtemporary Math., vol. 21, American Mathematical Society, Rhode Island, 1983, pp. 179-187.

[27] B. E. Rhoades, S. L. Singh, and C. Kulshrestha, Coincidence theorems for some multivalued mappings, Int. J. Math. Math. Sci. 7 (1984), no. 3, 429-434.

[28] K. P. R. Sastry and S. V. R. Naidu, Fixed point theorems for generalised contraction mappings, Yokohama Math. J. 28 (1980), 15-29.

[29] S. L. Singh, K. S. Ha, and Y. J. Cho, Coincidence and fixed points of nonlinear hybrid contractions, Int. J. Math. Math. Sci. 12 (1989), 247-256.

[30] S. L. Singh and S. N. Mishra, Nonlinear hybrid contractions, J. Natur. Phys. Sci. 5/8 (1991/1994), 191-206.

[31]_ On general hybrid contractions, J. Austral. Math. Soc. Ser. A 66 (1999), 244-254.

[32] R. E. Smithson, Fixed points for contractive multifunctions, Proc. Amer. Math. Soc. 27 (1971), 192-194.

[33] D. H. Tan and N. A. Minh, Some fixed-point theorems for mappings of contractive type, Acta Math. Vietnam. 3 (1978), no. 1, 24-42.

S. L. Singh: Department of MAthematics, Gurukula Kangri Vishwavidyalaya, HARDWAR 249404, INDIA

E-mail address: mathgkv@tatpar.com

S. N. Mishra: Department of Mathematics, University of Transkei, Umtata 5100, SOUTH AFRICA

E-mail address: mishra@getafix.utr.ac.za 


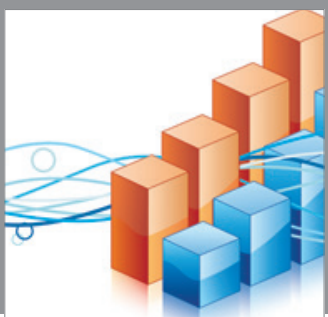

Advances in

Operations Research



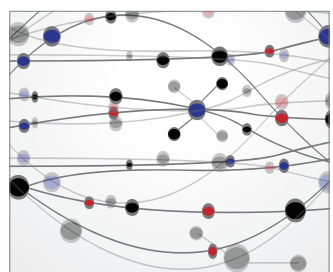

\section{The Scientific} World Journal
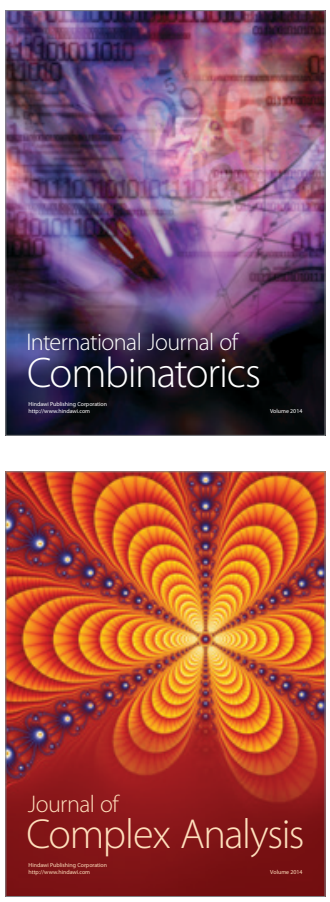

International Journal of

Mathematics and

Mathematical

Sciences
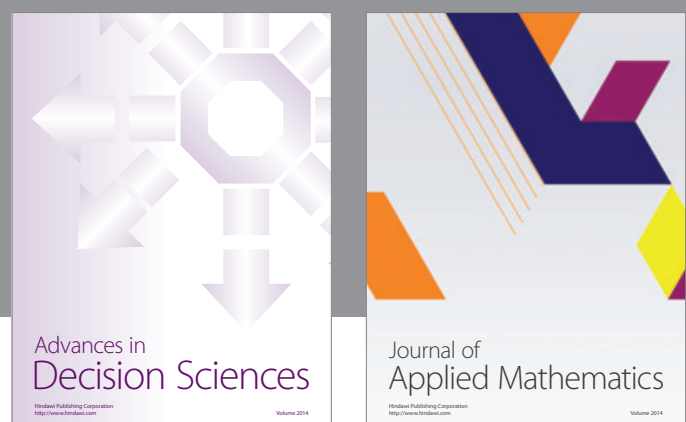

Journal of

Applied Mathematics
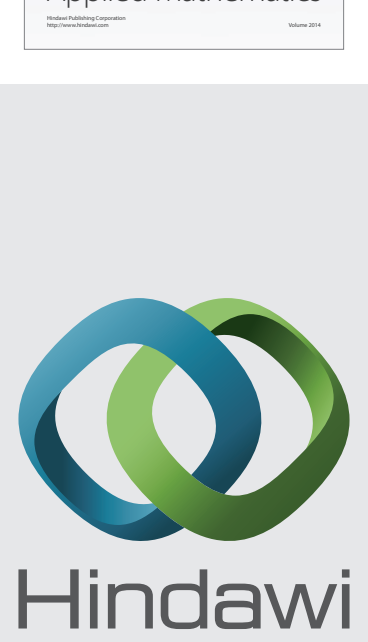

Submit your manuscripts at http://www.hindawi.com
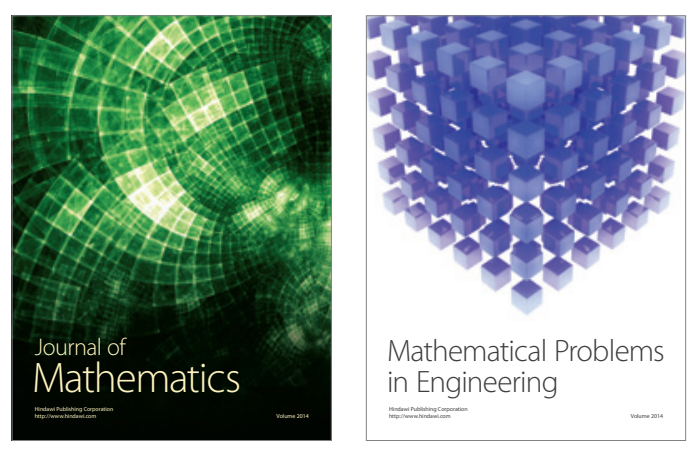

Mathematical Problems in Engineering
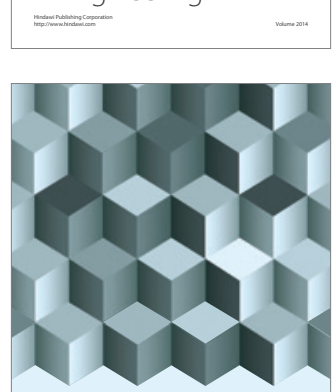

Journal of

Function Spaces
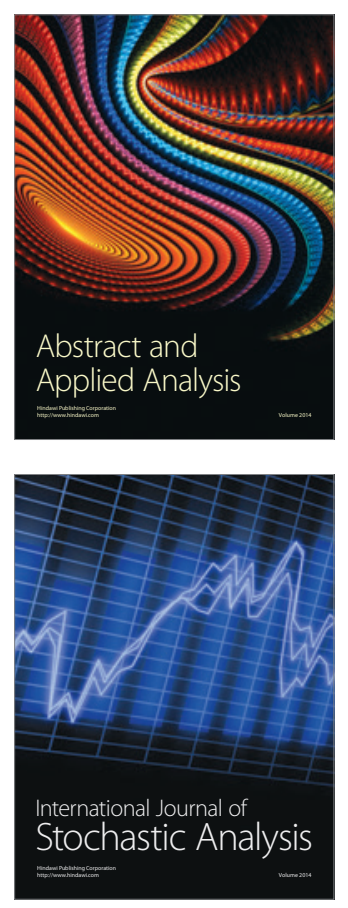

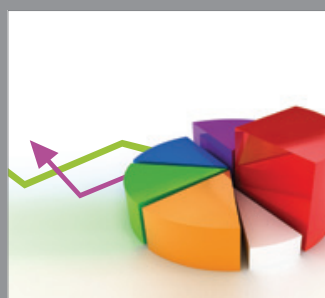

ournal of

Probability and Statistics

Promensencen
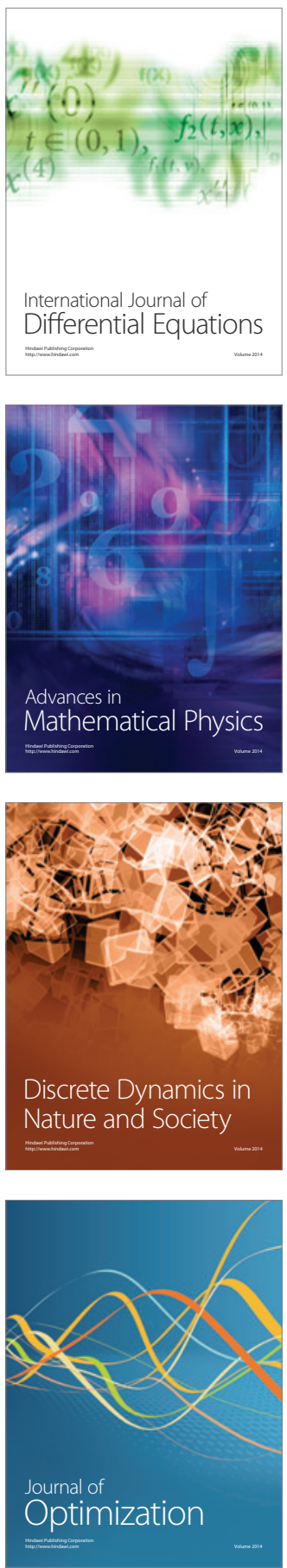